See discussions, stats, and author profiles for this publication at: https://www.researchgate.net/publication/329495404

\title{
ERCR TV: Ensemble of Random Committee and Random Tree for Efficient Anomaly Classification Using Voting
}

Conference Paper · April 2018

DOI: $10.1109 / 12$ CT.2018.8529797

CITATIONS

13

5 authors, including:

Niranjan Appaswamy

Gokaraju Rangaraju Institute of Engineering \& Technology

15 PUBLICATIONS 36 CITATIONS

SEE PROFILE

A Nitish $\mathrm{A}$

4. University of Mysore

4 PUBLICATIONS 22 CITATIONS

SEE PROFILE

Some of the authors of this publication are also working on these related projects:

Visualization and treatment of outliers View project

Efficient Multimedia Data Transfer Techniques for Mobile Cloud Computing View project
READS

530

Nutan D H

University Visweshwaraya College of Engineering, Bangalore University 1 PUBLICATION 13 CITATIONS

SEE PROFILE

P. Deepa Shenoy

University Visvesvaraya College of Engineering

178 PUBLICATIONS 1,289 CITATIONS

SEE PROFILE 


\title{
ERCRTV: Ensemble of Random Committee and Random Tree for Efficient Anomaly Classification using Voting
}

\author{
Niranjan A, Nutan D H, Nitish A, P Deepa Shenoy and Venugopal K R \\ Department of Computer Science and Engineering, \\ University Visvesvaraya College of Engineering, Bangalore University, \\ Bangalore, India. \\ a.niranjansharma@gmail.com
}

\begin{abstract}
Anomaly Detection is widely used in applications related but not limited to intrusion detection, fault detection, fraud detection, health monitoring systems and many other places. The overall efficiency of these applications depends on the classification algorithm that is chosen. An efficient classification algorithm can thus greatly improve the accuracy of these applications. This paper proposes a hybrid approach involving Random Committee and Random Tree techniques for anomaly classification, resulting in most encouraging accuracy values. The proposed scheme is followed after the preprocessing phase that involves Feature Selection using Correlated Feature Selection (CFS) algorithm with the Best-First Search technique.
\end{abstract}

Keywords-Random Tree; Random Committee; Anomaly Detection; Voting; Outlier Detection

\section{INTRODUCTION}

Anomaly detection [1] in the context of machine learning may be defined as the process of detecting data points in data that cannot fit well with the rest of the data. For this reason, Anomaly Detection is widely used in applications related but not limited to intrusion detection, fault detection, fraud detection, health monitoring systems and many other places. Anomaly detection is performed using one of the following three techniques:Unsupervised anomaly detection, supervised anomaly detection and Semi-supervised anomaly detection.

When detection of an anomaly is performed in an unlabeled test data set with the assumption that the majority of the instances in the data set are normal then is referred to as Unsupervised anomaly detection, while done on a data set that has been labeled as normal and abnormal and that involves training a classifier, it is referred to as Supervised Anomaly Detection. Semi-supervised anomaly detection techniques on the other hand involves the construction of a model representing normal behavior from a given normal training data set, and then testing the likelihood of a test instance to be generated by the learnt model.

The overall efficiency of these applications depends on the classification algorithm that is chosen. An efficient classification algorithm can thus greatly improve the accuracy of these applications. The metrics that can be used to measure the efficiency are Prediction Accuracy, Receiver Operating Characteristic (ROC) Area and Build time. They are in turn based on True Positive Rate (TPR) and False Positive Rate
(FPR). TPR is the measure of how correctly an anomalous instance is classified into an anomalous category while False Positive Rate (FPR) is the measure of incorrectness in classification. ROC is a graphical notation of illustrating the ability of a binary classifier system by varying its threshold. It is created by plotting TPR (Y-axis) against FPR (X-axis) at various threshold values. Build time is the time taken to train the given model. We expect the value of TPR to be the largest while the FPR and Build time values to be the least.

AIM: The current work aims at providing high detection accuracy with a significant reduction in FPR. This is achieved by selecting various classifying machine learning algorithms with better individual performances and their combinations (ensemble).

There are mainly four ensemble classifiers: Bagging, Boosting, Voting and Stacking. Bagging technique involves drawing $t$ random instances from a training set of size $t$ using a distribution that is uniform and learning these $t$ instances, and repeating the process many times. The instances that are drawn will contain few duplicates and few omissions in comparison with the original training set. Each repetition results in the generation of one classifier. After constructing several classifiers in this manner, the final prediction is determined based on a vote of the predictions of each classifier. Boosting is almost similar to bagging, except that the performance of the machine learning algorithm is continuously monitored and forces it to focus more on instances that have not been properly learnt. After several rounds of this process, the final prediction is done by considering votes having different weights based on the predictions of each classifier. The weights are assigned in proportion to each classifier's accuracy on its training set. Voting involves creating a number of sub-models and the predictions of all sub-models are combined in some way, such as by obtaining the mean of all the predictions, allowing each sub model to vote on what the final prediction should be. Stacking on the other hand unlike Bagging and Boosting, involves training an algorithm that is capable of combining the predictions of different learning algorithms. Each of the learning algorithms is initially trained using the available data, and finally the combiner algorithm is trained using the predictions of the each learning algorithm as additional inputs before making the final prediction. StackingC a variation of stacking has a better Accuracy Ratio and lesser run times. The StackingC algorithm available in WEKA uses Linear 
Regression as the default Meta Classifier. Machine learning or predictive modeling is concerned with minimizing the error of a model or making the most accurate predictions possible. Linear Regression in the context of Machine Learning may be defined as a representation of a linear equation that combines a specific set of numeric input values (x) to predicted numeric output (y). The current work provides an efficient ensemble approach for the better classification of the anomalous content in the data set through Voting.

TP and FP rates of different machine learning algorithms available in WEKA on NSL-KDD and KDD99 data sets were determined and our experiments proved that an ensemble of Random Committee and Random Tree algorithms through Voting, yields better performance results. The NSL-KDD and KDD99 data sets both contain 41 attributes, unfolding various network data flow features. The 42nd attribute is a class label that indicates whether the instance is normal or anomalous.

This work was taken up to achieve the following objectives:

- Application of different classifier algorithms to determine the most efficient classifiers in terms of Prediction Accuracy, ROC and Build time.

- Selection of the most relevant features from the data set for dimensionality reduction and faster runtime using the best Feature Selection Algorithm.

- To use a Hybrid Model of better Classification Algorithms to further enhance the performance of classification in terms of Prediction Accuracy.

The rest of the paper is organized as follows: Section II summarizes the related work carried out in the current domain while Section III presents the proposed ERCRTV model. Experimental Results are discussed in Section IV and Conclusion forms Section V of the paper.

\section{RELATED WORK}

In TDTC technique [2], the authors have proposed a novel approach to detect intrusions in IoT Backbone Networks through two-layer dimensionality reduction to address limitations that occur due to dimensionality which results in making wrong decisions while increasing computational complexity of the classifier. The Two-Layer dimensionality reduction is carried out by typically making use of Component Analysis and Linear Discriminate Analysis, followed by a Two-Tier classification through Naïve Bayes and Certainty Factor variation of the K-Nearest Neighbor techniques. Their work achieves a detection accuracy of 84.82 on the $20 \%$ of NSL-KDD train set.

The authors of [3] have proposed an anomaly-based detection technique for log analysis that makes use of data mining techniques. They have used Apache Hadoop to facilitate parallel processing of large data sets. Detection of new types of breaches without human intervention is achieved through dynamic rule creation. Their approach keeps the error rates below $10 \%$.
In [4], the authors have proposed a two-level ensemble model employing Boosting and Random Subspace Model (RSM) after applying PSO-based feature selection on the data set. Their model outperforms previous detection models in terms of accuracy and false positive rate (FPR).

The authors in [5] have proposed the use of Robust Regression technique in order to show the impact of detection of outliers in the training data set. They further prove that this model outperforms the ordinary Linear Regression technique which has been considered in the previous works. They show that their model is more reliable when compared to the ordinary Linear Regression technique especially for bursty network traffic and pervasive network attacks.

The paper proposed by the authors of [6] outlines a Proactive Anomaly Detection Ensemble approach in order to anticipate anomalies or abnormal patterns within a data set in a very timely manner. Proactive anomaly detection provides the flexibility of discovering anomalies before their occurrence through the use of weighted anomaly window as ground truth for training the model, which prioritizes early detection. Various strategies are explored for generating ground truth windows. Results have shown that ADE provides an improvement of at least $10 \%$ in earliest detection score compared to each individual technique across all data sets considered.

\section{PROPOSED ERCRTV}

The whole process, performed on WEKA simulator is divided into two phases namely, Preprocessing and Classification. The Preprocessing phase involves Feature Selection, since not all attributes in the data set are necessary for the analysis and it also results in dimensionality reduction, ensuring better performance. Phase two involves the application of an ensemble of Random Committee and Random Tree machine learning techniques for classification of the samples using Voting. Algorithm listing of the proposed ERCRTS is shown in Algorithm 1.

Algorithm 1: An Ensemble of Random Committee and Random Tree for Efficient classification of the samples using Voting (ERCRTV)

\section{1: while True do \\ 2: $\quad$ Load the KDD99/ NSL KDD Data Set \\ 3: $\quad$ Select features using CFS Subset Eval Feature Selection Algorithm with Best-First Search method \\ 4: $\quad$ Ensemble Random Committee and Random Tree using Voting for the classification of samples.}

\section{5: end while}

\section{A System Model of the proposed ERCRTV}

Machine learning algorithms are the mostly used techniques for the implementation of Intrusion Detection System as some of these techniques provide the highest classification accuracy 
with least false positive rates. With the aim of constructing an Anomaly Detection model having high classification accuracy,

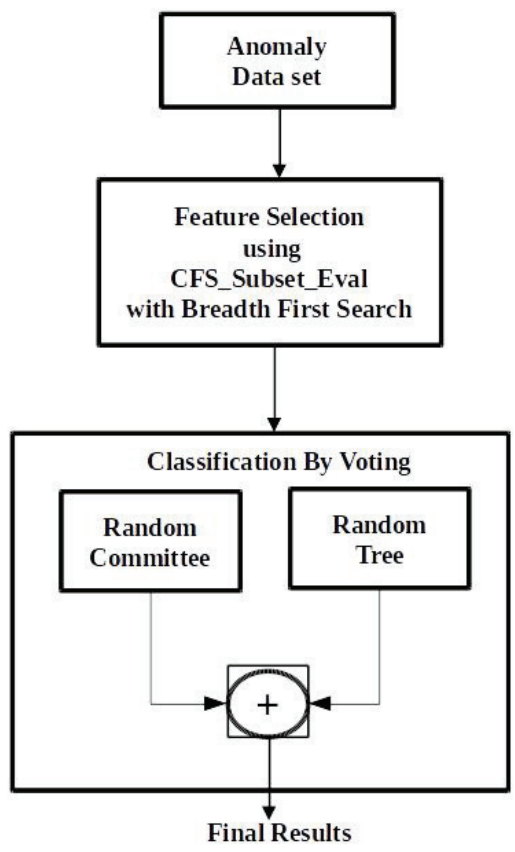

Fig.1. System model for the proposed ERCRTV

low or nil false positives and low model building time, we apply an ensemble of Random Committee and Random Tree machine learning techniques for classification of the samples using Voting to an Anomaly Detection System. Random Tree and Random Committee are chosen through the Voting feature available on WEKA for the classification of the samples. Experimental results show that the proposed model exhibits all the desired characteristics. Fig 1 summarizes the model of the proposed system.

\section{EXPERIMENTAL RESULTS AND DISCUSSION}

The Data sets that were chosen for our experiments are NSL-KDD and KDDCup99. The NSL-KDD has both train and test sets each having 125973 and 22544 instances respectively. The proposed model was initially trained using the train set and was then tested using the test set. To measure the performance metrics a Ten-fold cross validation was performed. The performance of the proposed model was later validated by using the KDDCup99 data set. Keeping the extremely huge size of this data set with 494020 instances in mind, it was decided to split this up into train and test sets in the ratio of 60:40 with the train set getting a share of 296412 while the test set getting 197608 instances.

As a part of the pre-processing phase, the data set is loaded into the simulator and is preprocessed to remove less significant attributes. Correlated Feature Selection (CFS) Subset attribute evaluator accompanied by the default BestFirst Search method resulted in a Feature subset comprising of only 8 significant features out of a total of 41 features while all other Feature Selection Techniques resulted in larger feature subset as listed in Table 1. The main reason behind CFS selecting least number of features is because; it considers correlation between features for the selection. The CFS removes all possible redundant and irrelevant features with no correlation between them thereby significantly improving the execution speed. The CFS technique generates decision rules by using Random Tree Classifier [7].

Various classification algorithms with the reduced Feature set are tested and the ten-fold cross validation results are tabulated in Table 2. Noticing higher precision rates along with lesser build time obtained for Random Committee and Random Tree, ensemble of the same using Voting and Stacking was performed and the results as listed in Table 3 were determined for the same. Though the Prediction Accuracy of Stacking scheme is almost closer to the Voting results, the Build Time of the same is huge as compared to Voting. Hence Voting was chosen as the ensemble technique for the proposed model.

Table 1 Performance of Different Feature Selection Techniques

\begin{tabular}{lcc}
\hline & Number of & \\
Feature Selection & Selected & Features \\
Algorithm & Features & Selected
\end{tabular}

$\begin{array}{lcc}\text { CFS_Subset_Eval } & 8 & \text { F12,F26,F29, } \\ \text { ChiSquaredAttribureEval } & 41 & \text { F30,F37 } \\ \text { ClassifierSubsetEval } & \text { None } & \text { F1-F41 } \\ \text { ConsistencySubsetEval } & \text { None } & \text { Nil } \\ \text { Filtered Attribute Eval } & 41 & \text { F1-F41 } \\ \text { Gain Ratio Attribute } & & \\ \text { Eval } & 41 & \text { F1-F41 } \\ \text { Info Gain } & 41 & \text { F1-F41 } \\ \text { One R Attribute Eval } & 41 & \text { F1-F41 } \\ \text { Relief Attribute Eval } & \text { None } & \text { Nil } \\ \text { Symmetrical_Uncer } & 41 & \text { F1-F41 } \\ \text { Attribute Eval } & & \\ \text { Wrapper Subset Eval } & \text { None } & \text { Nil }\end{array}$

Table 2 Ten-fold cross validation results of different Classification Techniques

\begin{tabular}{lcccc}
\hline & \multicolumn{2}{c}{ NSL-KDD (Full Train Set) } & \multicolumn{2}{c}{ KDD (Train-60\%) } \\
\hline Classifier algorithm & $\begin{array}{c}\text { Prediction } \\
\text { Accuracy }\end{array}$ & $\begin{array}{c}\text { Build } \\
\text { Time } \\
\text { (in sec) }\end{array}$ & $\begin{array}{c}\text { Prediction } \\
\text { Accuracy }\end{array}$ & $\begin{array}{c}\text { Build } \\
\text { Time } \\
\text { (in sec) }\end{array}$ \\
\hline Random Tree & 99.6 & 0.97 & 100 & 2.36 \\
RandomCommittee & 99.6 & 10.64 & 100 & 24.08 \\
Random Forest & 99.6 & 99.91 & 100 & 268.8 \\
Decision Table & 99.4 & 19.42 & 99.8 & 34.78 \\
Random Subspace & 99.2 & 12.03 & 99.6 & 25.03 \\
Bayes Net & 96.5 & 1.53 & 99.8 & 2.3 \\
One R & 96.5 & 0.25 & 97.9 & 0.42 \\
ADABoostM1 & 93.4 & 5.11 & 93.8 & 11.42 \\
Naïve Bayes & 89.4 & 0.38 & 93.8 & 0.64 \\
\hline
\end{tabular}


Table 3 Performance of different Ensemble Techniques

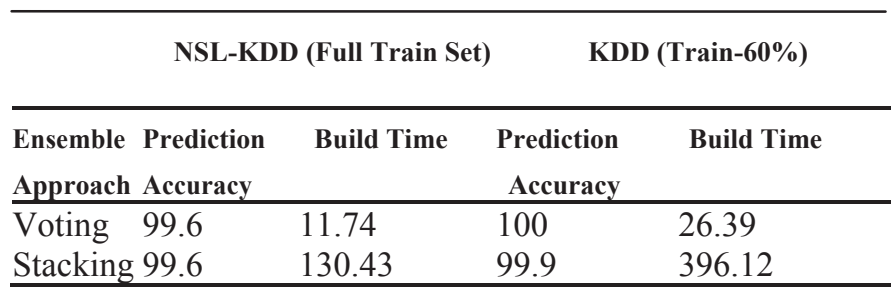

Random committee involves the construction of a number of Base classifiers using unique random number seed values and the final classification result is provided by computing the average of the predictions generated by the individual base classifiers. Random Tree on the other hand is a decision tree built on a random subset of attributes. A decision tree is a group of nodes and their branches. A node of a decision tree represents a test on an attribute while each branch represents its outcome. The leaves of a decision tree denote the final decision taken after computing all attributes in the form of class labels. The path covered from root to a leaf forms a classification rule.

The efficiency of classification could be attributed to the maximum true positive rates and minimum false positive rates. With respect to this context, we define 5 measures of classification process. Let $\mathrm{N}_{\text {norm }}$ and $\mathrm{N}_{\text {anom }}$ be the total number of normal and anomalous instances in a data set respectively. The number of normal instances classified as normal are denoted as $\mathrm{N}_{\text {norm } \rightarrow \text { norm }}(\mathrm{TP})$ and number of anomalous instances classified as anomalous are denoted as $\mathrm{N}_{\text {anom } \rightarrow \text { anom }}(\mathrm{TN})$. The number of normal instances misclassified as anomalous are denoted as $\mathrm{N}_{\text {norm } \rightarrow \text { anom }}$ (FP) and the number of anomalous instances misclassified as normal are denoted as $\mathrm{N}_{\text {anom } \rightarrow \text { norm }}$ (FN).

$$
\begin{aligned}
& \mathrm{TP}=\mathrm{N}_{\text {norm }} \rightarrow^{\text {norm }} / \mathrm{N}_{\text {norm }} \text { X } 100 \\
& \mathrm{TN}=\mathrm{N}_{\text {anom }} \rightarrow^{\text {anom }} / \mathrm{N}_{\text {anom }} \text { X } 100 \\
& \mathrm{FP}=\mathrm{N}_{\text {norm }} \rightarrow_{\text {anom }} / \mathrm{N}_{\text {norm }} \text { X } 100 \\
& \mathrm{FN}=\mathrm{N}_{\text {anom }^{\text {norm }}} / \mathrm{N}_{\text {anom }} \text { X } 100
\end{aligned}
$$

$$
\begin{aligned}
\text { Prediction Accuracy }= & \left(\mathrm{N}_{\text {norm }} \rightarrow^{\text {norm }}+N_{\text {anom }_{\rightarrow} \text { anom }}\right) \\
& /\left(N_{\text {norm }}+N_{\text {anom }}\right) X 100
\end{aligned}
$$

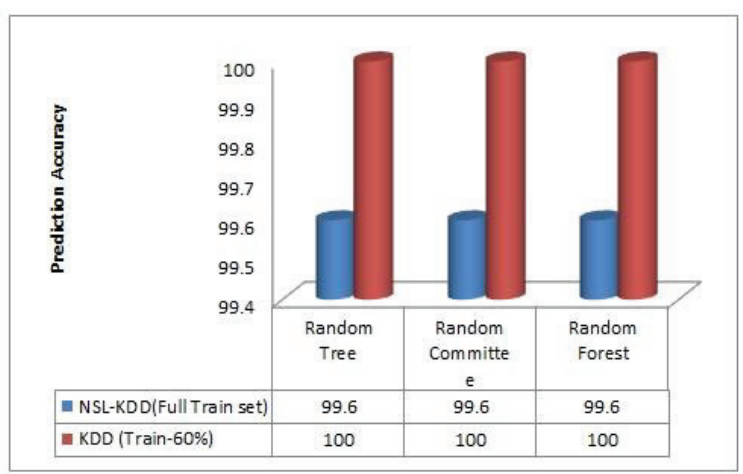

Fig.2. Prediction Accuracy of Classifiers

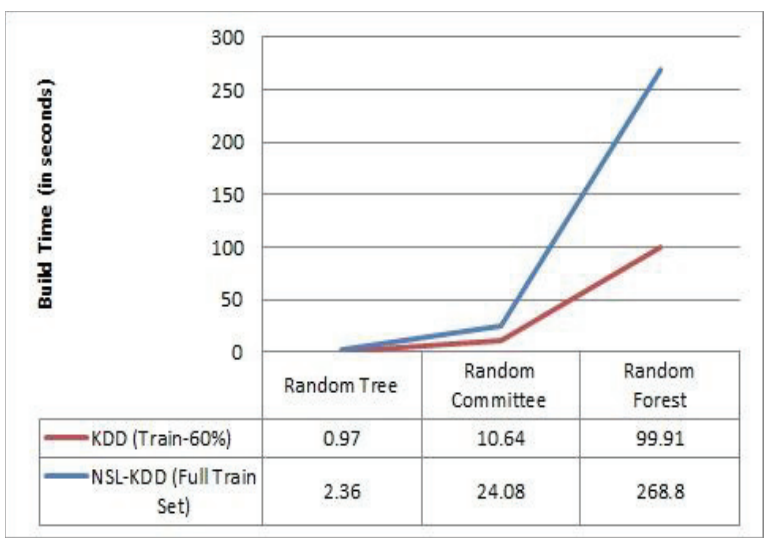

Fig.3. Build Time of Classifiers

It may be noticed from Fig 2 and Fig 3 that Random Tree and Random Committee classifiers perform better on both NSL-KDD and KDD99 data sets.

Fig 4 and Fig 5 illustrate the efficiency of Voting Ensemble Technique of Random Tree and Random Committee classifiers in terms of Prediction Accuracy and Build Time respectively.

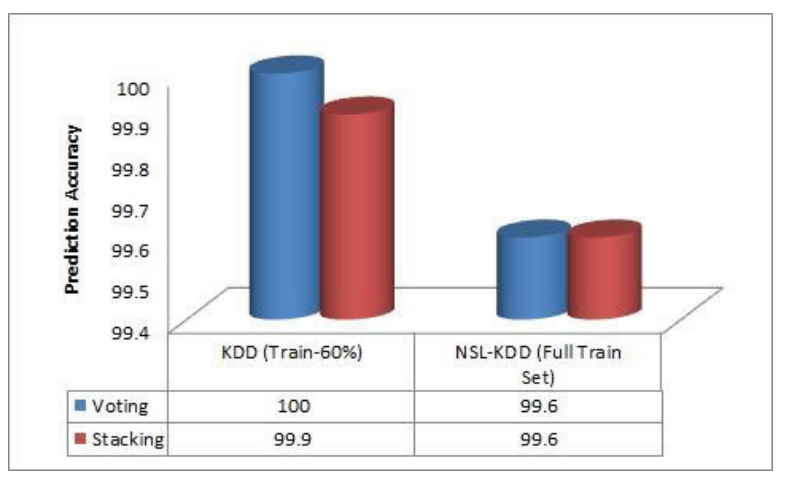

Fig.4. Prediction Accuracy of Ensemble techniques 


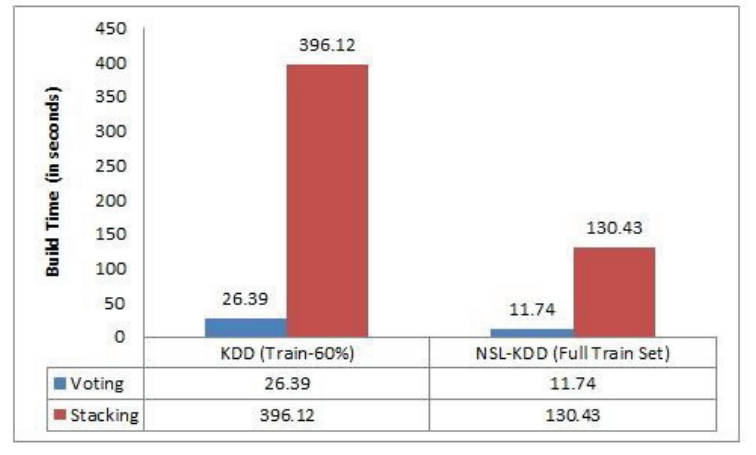

Fig.5. Build Time of Ensemble Techniques

\section{V.CONCLUSION}

Feature selection [8] is applied as a part of the pre-processing [9] phase on the data set to reduce dimensionality and to remove all the redundant and irrelevant features. Out of the 41 Features that are present in NSL KDD and KDDcup99, only 8 Features are chosen by the CFS algorithm. After the elimination of the less significant features in this manner, Voting feature available in WEKA was utilized for combining the Random Committee and Random Tree Classification Techniques. A tenfold cross validation was also applied for the proposed classification model. The experimental results prove that prediction accuracy of the proposed model is better compared to the Stacking technique while consuming lesser Build time values. The proposed model is required to be tested on other data sets as well and total build time required to carry out classification has to be further reduced.

\section{REFERENCES}

[1] Niranjan A, Nitish A, P Deepa Shenoy and Venugopal K R, "Security in Data Mining-a Comprehensive Survey", Global Journal of Computer Science and Technology, vol. 16, no. 5, 2017, pp. 52-73.

[2] H Haddad Pajouh, R Javidan, R Khayami, D Ali and K K R Choo, "A Two-layer Dimension Reduction and Two-tier Classification Model for Anomaly-Based Intrusion Detection in IoT Backbone Networks", IEEE Transactions on Emerging Topics in Computing, vol. pp, no. 99, pp. 111.

[3] Jakub Breier and Jana Branisov, "Anomaly Detection from Log files using Data Mining Techniques", Proceedings of Conference on Information Science and Applications (Springer), vol. 339, 2015, pp. 449-457.

[4] Bayu Adhi Tama, Akash Suresh Patil, Kyung-Hyune Rhee "An Improved Model of Anomaly Detection using

Two-level Classifier Ensemble", Proceedings of 12th Asia Joint Conference on Information Security, 2017.

[5] Ziyu Wang, Jiahai Yang, Zhang ShiZe, Chenxi Li "Robust Regression for Anomaly Detection", Proceedings of IEEE ICC 2017 Communication and Information Systems Security Symposium, 2017.

[6] Teodora Sandra Buda1, Haytham Assem1 and Lei Xu1 "ADE: An Ensemble Approach for Early Anomaly Detection", Integrated Network and Service Management (IM), 2017 IFIP/IEEE Symposium, 2017.

[7] Aman Gupta and Pranita Jain, "A Map Reduce Hadoop Implementation of Random Tree Algorithm based on Correlation Feature Selection", International Journal of Computer Applications, vol. 160, no. 5, 2017, pp. 41-44.

[8] Srinivasa K G, Amrinder Gill, A O Thomas, Venugopal K R and L M Patnaik, "Generic Feature Extraction for Classification using Fuzzy c Means Clustering", In IEEE Proceedings of Third International Conference on Intelligent Sensing and Information Processing, IEEE Computer Society Press, pp. 33-38, Bangalore, India, December, 2005.

[9] Asha S Manek, Samhitha M R, Shruthy S, Veena H Bhat, P Deepa Shenoy, M. Chandra Mohan, Venugopal K R, L M Patnaik "RePID-OK: Spam Detection using Repetitive Pre-processing", IEEE CUBE 2013 Conference, ISBN : 978-1-4799-2234-5, pp. 144-149, November 15-16, 2013. 